\title{
Diarylsulfonylurea Compound ILX-295501
}

National Cancer Institute

\section{Source}

National Cancer Institute. Diarylsulfonylurea Compound ILX-295501. NCI Thesaurus.

Code C1632.

ILX-295501 is a novel sulfonylurea compound that has demonstrated in-vivo antitumor activity against a broad spectrum of solid tumors. 